\title{
The Multidimensional Darboux Transformation
}

\author{
Artemio González-López ${ }^{\dagger}$ \\ Departamento de Física Teórica II \\ Universidad Complutense \\ Madrid \\ SPAIN 28040 \\ artemio@ciruelo.fis.ucm.es
}

\author{
Niky Kamran $\ddagger$ \\ The Fields Institute \\ 222 College Street \\ Toronto, Ontario \\ CANADA M5T 3J1 \\ nkamran@math.mcgill.ca
}

\begin{abstract}
A generalization of the classical one-dimensional Darboux transformation to arbitrary $n$-dimensional oriented Riemannian manifolds is constructed using an intrinsic formulation based on the properties of twisted Hodge Laplacians. The classical two-dimensional Moutard transformation is also generalized to non-compact oriented Riemannian manifolds of dimension $n \geq 2$. New examples of quasi-exactly solvable multidimensional matrix Schrödinger operators on curved manifolds are obtained by applying the above results.
\end{abstract}

\footnotetext{
$\dagger$ Supported in part by DGICYT Grants PB92-0197 and PB96-019\%.

$\ddagger$ Supported in part by an NSERC Grant. On sabbatical leave from the Department of Mathematics and Statistics, McGill University, Montreal, QC, H3A 2K6.
}

December 3, 2013 


\section{Introduction}

Our purpose in this paper is to define and study the properties of a broad generalization to $n$ dimensions of the classical Darboux transformation for Sturm-Liouville operators on the line. Our approach will stem from a geometric generalization of the basic intertwining relations underlying the classical Darboux transformation to the context of certain twisted Laplacians acting on the exterior algebra of an oriented Riemannian manifold.

Let us begin by recalling the essentials of the one-dimensional Darboux transformation, $[\mathbf{4}],[\mathbf{3}]$. Consider a Sturm-Liouville operator $h$, given by

$$
h=-\frac{d^{2}}{d x^{2}}+V(x)
$$

and let $e^{-\chi}$ be a nowhere vanishing eigenfunction of $h$ with eigenvalue $E_{0}$,

$$
\left(h-E_{0}\right) e^{-\chi}=0 .
$$

The classical Darboux transformation associates to $h$ the Sturm-Liouville operator $\widetilde{h}$ defined by

$$
\widetilde{h}=-\frac{d^{2}}{d x^{2}}+\widetilde{V}(x)
$$

where

$$
\widetilde{V}=V+2 \chi^{\prime \prime}
$$

It is straightforward to verify that the operators $h$ and $\widetilde{h}$, shifted by $E_{0}$, can be factorized in the following way,

$$
h-E_{0}=Q^{+} Q^{-}, \quad \widetilde{h}-E_{0}=Q^{-} Q^{+},
$$

where $Q^{+}$and $Q^{-}$are first-order differential operators defined by

$$
Q^{+}=-\frac{d}{d x}+\chi^{\prime}, \quad Q^{-}=\frac{d}{d x}+\chi^{\prime}
$$

The operators $h-E_{0}, \widetilde{h}-E_{0}, Q^{+}$and $Q^{-}$are therefore related by the intertwining relations

$$
\left(h-E_{0}\right) Q^{+}=Q^{+}\left(\widetilde{h}-E_{0}\right), \quad Q^{-}\left(h-E_{0}\right)=\left(\widetilde{h}-E_{0}\right) Q^{-} .
$$

We thus obtain a simple relation between the eigenfunctions (formal or $\mathrm{L}^{2}$ ) of $h$ and those of its Darboux transform $\widetilde{h}$. Indeed, if $\psi$ is a formal eigenfunction of $h$ with eigenvalue $E \neq E_{0}$, then it follows immediately from the above intertwining relations that $Q^{-} \psi$ will be a formal eigenfunction of $\widetilde{h}$ with the same eigenvalue. Conversely, if $\tilde{\psi}$ is a formal eigenfunction of $\widetilde{h}$ with eigenvalue $E \neq E_{0}$, then $Q^{+} \psi$ will be a formal eigenfunction of $h$ with eigenvalue $E$. It is not difficult to show that this correspondence also holds at the level of the $\mathrm{L}^{2}$ eigenfunctions of $h$ and $\widetilde{h}$, so that the Darboux transformation establishes a correspondence between bound states of $h$ and $\widetilde{h}$. 
It is well-known that the Darboux transformation plays an important role in the theory of soliton solutions of integrable evolution equations and in the method of inverse scattering, [5]. It also provides a powerful method for generating new exactly or quasi-exactly solvable one-dimensional potentials from known ones [13]. The Darboux transformation also appears as a basic tool in the theory of special functions through the factorization method of Infeld and Hull, [10]. The problem of extending the Darboux transformation to the case of multi-dimensional differential operators is therefore of considerable interest.

There are at least two natural candidates for what could be called a Darboux transformation in two dimensions, namely the Laplace, [6], and Moutard, [12], transformations. The Moutard transformation is perhaps closer in spirit to the classical Darboux transformation, since it is based on intertwining relations analogous to the ones given above for the Darboux transformation. This will be made explicit in Section 5. On the other hand, the Laplace transformation plays a significant role in the realm of integrable systems. For example, it naturally gives rise to the Lax representation for the $A_{n}$ Toda lattice, [14]. It is also at the basis of some important recent work on exactly solvable periodic two-dimensional Schrödinger operators, [15]. In any case, both the Laplace and Moutard transformations preserve the class of linear elliptic second-order differential operators in the plane. However, unlike the one-dimensional Darboux transformation, they both suffer from the major limitation that they yield only one eigenfunction for the transformed operator, $[\mathbf{1 5}]$, the reason being that they do not incorporate the spectral parameter $E$. Let us briefly illustrate this difficulty in the case of the Laplace transformation. The conclusion is analogous for the Moutard transformation. Consider the two-dimensional Schrödinger equation given by

$$
\left(-\frac{\partial^{2}}{\partial z \partial \bar{z}}+V(x, y)\right) \psi=E \psi
$$

in terms of complex coordinates $z=\frac{1}{2}(x+i y), \bar{z}=\frac{1}{2}(x-i y)$. Under the Laplace transformation, the wave function $\psi$ gets mapped to the wave function $\hat{\psi}$ defined by

$$
\hat{\psi}=\frac{\partial \psi}{\partial \bar{z}}
$$

and it is easily verified that the transformed wave function $\hat{\psi}$ satisfies the following Schrödinger equation for a particle in a magnetic field

$$
\left[-\frac{\partial^{2}}{\partial z \partial \bar{z}}+\frac{\partial \log (V-E)}{\partial \bar{z}} \frac{\partial}{\partial z}+V-E\right] \hat{\psi}=0 .
$$

The explicit dependence of the coefficient of $\partial / \partial z$ on $E$ illustrates our point. There is also a drawback which is specific to the Laplace transformation, namely that its geometric generalization to $n$ dimensions, $[\mathbf{1 1}]$, applies to a class of highly overdetermined systems which bear no relation to any natural spectral problem, although they are of course interesting in their own right. Another essential limitation of the Laplace and Moutard transformations is that they are only defined for flat Laplacians expressed in Cartesian coordinates, whereas many of the Schrödinger operators arising by symmetry reduction involve curved 
Laplacians in very general coordinate systems. One would therefore like to have a multidimensional Darboux transformation which is defined in a covariant way, which allows for curvature of the underlying manifold and which includes the spectral parameter in a natural way.

An indication on how to proceed is suggested by the work of Andrianov, Borisov and Ioffe, $[\mathbf{1}]$. In their scheme, one starts from a Schrödinger operator $h$ in the Euclidean plane, expressed in Cartesian coordinates, and one constructs, starting from a nowhere vanishing eigenfunction of $h$, the Moutard transform $\widetilde{h}$ of $h$, and a two-by-two matrix Schrödinger operator $H$ which splits into the sum of two operators $H^{(1)}$ and $H^{(2)}$. The intertwining relations between $h, \widetilde{h}, H^{(1)}$ and $H^{(2)}$ imply that $h$ and $H^{(1)}$ have the same eigenvalues except possibly for the zero eigenvalue, and similarly for $\widetilde{h}$ and $H^{(2)}$. (This construction was later generalized in [2] to flat $n$-dimensional Euclidean space, still using Cartesian coordinates in an essential way.) After a careful analysis of this scheme, we conclude that the operators $h, H$ and $\widetilde{h}$ introduced in [2] can in fact be re-expressed as twisted flat Laplacians acting on 0 -forms, 1-forms and 2-forms, the latter being identified with 0-forms by means of the Hodge operator for the underlying two-dimensional flat Euclidean metric. The intertwining relations between $h, \widetilde{h}, H^{(1)}$ and $H^{(2)}$ will now follow immediately from elementary properties of the twisted differentials and codifferentials.

Starting from this observation, we succeed in our paper to construct a fully covariant and coordinate-free multidimensional generalization of the classical Darboux transformation, valid on an arbitrary curved $n$-dimensional oriented Riemannian manifold. Our $n$ dimensional Darboux transformation relates, via intertwining relations involving twisted differentials and codifferentials, the spectra and eigenfunctions of a string of $n+1$ twisted Laplacians acting on $k$-forms for $0 \leq k \leq n$. It is noteworthy that these are the twisted Laplacians which were used by Witten in his proof of the Morse inequalities based on ideas from supersymmetry, [17]. When expressed in any coordinate system, these twisted Laplacians take the form of matrix Schrödinger operators acting on the $\left(\begin{array}{l}n \\ k\end{array}\right)$ components of a $k$-form. In the special case where the underlying manifold is flat Euclidean space in Cartesian coordinates, our multidimensional Darboux transformation reproduces the classical Darboux transformation and the scheme of [1] and [2].

Our paper is organized as follows. In Section 2, we define the twisted versions of the differential, the codifferential and the Laplacian on forms. When expressed in local coordinates, the latter will correspond to scalar and matrix Schrödinger operators on curved Riemannian manifolds. In Section 3, we first derive the basic intertwining relations defining our multidimensional Darboux transformation and give their spectral interpretation. This generalization of the Darboux transformation is valid for twisted Laplacians on an arbitrary $n$-dimensional oriented Riemannian manifold. We then derive the local coordinate expressions of the resulting matrix Schrödinger operators in terms of the seed eigenfunction for the original scalar Hamiltonian and the Riemann curvature of the background metric. The connection between the spectra of our matrix Hamiltonians admits an interesting interpretation in terms of supersymmetry, cf. $[\mathbf{1 6}],[\mathbf{1 7}],[\mathbf{2}]$, which we briefly recall. In Section 4, we show that our multidimensional Darboux transformation reduces to the classical Darboux transformation on the line and to a covariant coordinate-free generalization to curved oriented Riemannian manifolds of the scheme of references $[\mathbf{1}]$ and $[\mathbf{2}]$ in 
two dimensions. In Section 5, we derive an $n$-dimensional generalization of the Moutard transformation which applies to all twisted Laplacians. The spectral interpretation of the multi-dimensional Moutard transformation is significantly more limited than that of the multidimensional Darboux transformation, because it only applies to the zero modes of the twisted Laplacians. Finally, in Section 6 we obtain new examples of multi-dimensional quasi-exactly or exactly solvable matrix Schrödinger operators of physical interest, of which very few seem to be known, by applying the multidimensional Darboux transformation to various quasi-exactly solvable planar Hamiltonians, [7].

\section{Twisted Laplacians and Schrödinger operators}

Our purpose in this section is to define a twisted version of the Laplacian on $k$-forms on an oriented Riemannian manifold. On scalar functions, this twisted Laplacian will correspond to a Schrödinger operator.

We start by setting up some notation. Let $M$ be an $n$-dimensional oriented Riemannian manifold, with metric $\left(g_{i j}\right)_{1 \leq i, j \leq n}$. We shall denote by $\Lambda^{k}(M)$ the vector space of differential $k$-forms on $M$, and by $\Lambda(M)=\bigoplus_{k=o}^{n} \Lambda^{k}(M)$ the exterior algebra of differential forms on $M$. In a local coordinate system $x=\left(x^{1}, \ldots, x^{n}\right)$ a $k$-form $\omega \in \wedge^{k}(M)$ will be written as

$$
\omega=\frac{1}{k !} \omega_{i_{1} \ldots i_{k}}(x) d x^{i_{1}} \wedge \ldots \wedge d x^{i_{k}}
$$

where $\omega_{i_{1} \ldots i_{k}}$ is an antisymmetric tensor. Here, and in what follows, we are using the summation convention.

Since $M$ is Riemannian and oriented, it admits a volume form $\mu \in \Lambda^{n}(M)$, which in a positively oriented coordinate system $x=\left(x^{1}, \ldots, x^{n}\right)$ can be written as

$$
\mu=\sqrt{g(x)} d x^{1} \wedge \ldots \wedge d x^{n}
$$

where

$$
g=\operatorname{det}\left(g_{i j}\right)
$$

The Riemannian metric on $M$ induces a scalar product on the exterior algebra of $M$ in a natural way. Namely, if $\alpha$ and $\beta$ are two $k$-forms with (antisymmetric) components $\alpha_{i_{1} \ldots i_{k}}$ and $\beta_{i_{1} \ldots i_{k}}$, respectively, one defines

$$
\langle\alpha, \beta\rangle=\frac{1}{k !} \alpha_{i_{1} \ldots i_{k}} \beta^{i_{1} \ldots i_{k}}=\frac{1}{k !} \alpha^{i_{1} \ldots i_{k}} \beta_{i_{1} \ldots i_{k}}
$$

where the functions

$$
\alpha^{i_{1} \ldots i_{k}}=g^{i_{1} j_{1}} \cdots g^{i_{k} j_{k}} \alpha_{j_{1} \ldots j_{k}}
$$

are the contravariant components of $\alpha$, and

$$
\left(g^{i j}\right)=\left(g_{i j}\right)^{-1}
$$


is the contravariant metric tensor. It is clear that the definition of the function $\langle\alpha, \beta\rangle$ does not depend on the local coordinates used; hence one defines the inner product $(\alpha, \beta)$ of the $k$-forms $\alpha, \beta \in \Lambda^{k}(M)$ by

$$
(\alpha, \beta)=\int_{M}\langle\alpha, \beta\rangle \mu .
$$

The inner product $(\alpha, \beta)$ will only be defined if the above integral is convergent; for instance, this will be the case if $\alpha$ and $\beta$ are compactly supported, or in particular if $M$ is a compact manifold.

Given a $k$-form $\beta \in \wedge^{k}(M)$, the Hodge star $* \beta \in \wedge^{n-k}(M)$ of $\beta$ is defined as usual as the unique $(n-k)$-form satisfying

$$
\alpha \wedge * \beta=\langle\alpha, \beta\rangle \mu, \quad \forall \alpha \in \bigwedge^{k}(M) .
$$

The Hodge star operator $*: \wedge^{k}(M) \rightarrow \wedge^{n-k}(M)$ has the following elementary properties, which we list here for future reference:

$$
\begin{aligned}
& \alpha \wedge * \beta=\beta \wedge * \alpha, \quad \alpha, \beta \in \bigwedge^{k}(M) \\
& * * \alpha=(-1)^{k(n-k)} \alpha, \quad \alpha \in \bigwedge^{k}(M) \\
& * \mu=1, \quad * 1=\mu .
\end{aligned}
$$

In local coordinates the components of the Hodge dual of a $k$-form $\alpha$ are given by

$$
(* \alpha)_{j_{k+1} \ldots j_{n}}=\frac{\sqrt{g}}{k !} \epsilon_{j_{1} \ldots j_{n}} \alpha^{j^{1} \ldots j^{k}}, \quad \alpha \in \bigwedge^{k}(M),
$$

where $\epsilon_{j_{1} \ldots j_{n}}$ is antisymmetric under permutations of its indices, and

$$
\epsilon_{12 \ldots n}=+1 .
$$

The Hodge star operator is used to define the codifferential $\delta$ of a $k$-form $\alpha$ by the formula

$$
\delta \alpha=(-1)^{n(k-1)+1} * d(* \alpha), \quad \alpha \in \bigwedge^{k}(M) .
$$

Thus, $\delta$ maps $\wedge^{k}(M)$ into $\wedge^{k-1}(M)$; in particular, $\delta=0$ on $\wedge^{0}(M)=C^{\infty}(M)$. In local coordinates, one can show that

$$
(\delta \alpha)_{i_{1} \ldots i_{k-1}}=-\nabla^{j} \alpha_{j i_{1} \ldots i_{k-1}},
$$

where $\nabla$ denotes the covariant derivative associated to the Riemannian metric. The odd looking sign in the definition of $\delta$ is not arbitrary, since with this choice of sign the operators $d$ and $\delta$ are the formal adjoint of one another with respect to the scalar product on $\Lambda(M)$,

$$
d_{k}^{\dagger}=\delta_{k+1}, \quad 0 \leq k \leq n-1 .
$$


It also follows immediately from its definition that $\delta$ is a coboundary operator, namely

$$
\delta^{2}=0 .
$$

Furthermore, the local exactness of $d$ (Poincaré's lemma) implies that $\delta$ is also locally exact: in other words, if $\delta \omega=0$ then there is an open neighborhood of every point of $M$ on which $\omega=\delta \alpha$, for some differential form $\alpha$.

The Laplacian $\Delta: \wedge(M) \rightarrow \wedge(M)$, defined by

$$
-\Delta=d \delta+\delta d,
$$

maps each space $\wedge^{k}(M)$ into itself for all $k=0,1, \ldots, n$. Moreover, from the elementary properties of the codifferential it follows that $-\Delta$ is (formally) self-adjoint and nonnegative:

$$
\begin{aligned}
& (\alpha,-\Delta \beta)=(-\Delta \alpha, \beta), \quad \alpha, \beta \in \bigwedge^{k}(M) \\
& (\alpha,-\Delta \alpha)=(d \alpha, d \alpha)+(\delta \alpha, \delta \alpha) \geq 0, \quad \alpha \in \bigwedge^{k}(M) .
\end{aligned}
$$

If $f \in \Lambda^{0}(M)$ is a smooth function on $M$, we obtain using (6) that

$$
\Delta f=-\delta d f=\nabla^{i} \nabla_{i} f=\frac{1}{\sqrt{g}} \frac{\partial}{\partial x^{i}}\left(\sqrt{g} g^{i j} \frac{\partial f}{\partial x^{j}}\right)
$$

is the classical Laplace-Beltrami operator on $\Lambda^{0}(M)$. If $\omega$ is a $k$-form, the components of $-\Delta \omega$ in a local coordinate system are given by the following expression:

$$
\begin{aligned}
(-\Delta \omega)_{i_{1} \ldots i_{k}}=- & \nabla^{j} \nabla_{j} \omega_{i_{1} \ldots i_{k}}+\sum_{q=1}^{k}(-1)^{q+1} R^{j}{ }_{i_{q}} \omega_{j i_{1} \ldots \widehat{i_{q}} \ldots i_{k}} \\
& +2 \sum_{1 \leq p<q \leq k}(-1)^{p+q+1} R^{j}{ }_{i_{p}}{ }^{l}{ }_{i_{q}} \omega_{j l i_{1} \ldots \widehat{i_{p} \ldots} \widehat{i_{q} \ldots i_{k}}} .
\end{aligned}
$$

In the above equation a hat over an index means that that index is to be omitted, the Riemann tensor $R_{j h l}^{i}$ is defined by

$$
R_{j h l}^{i}=\frac{\partial \Gamma_{j h}^{i}}{\partial x^{l}}-\frac{\partial \Gamma_{j l}^{i}}{\partial x^{h}}+\Gamma_{p l}^{i} \Gamma_{j h}^{p}-\Gamma_{p h}^{i} \Gamma_{j l}^{p}
$$

in terms of the connection coefficients $\Gamma_{j l}^{i}$ of the metric, and $R_{j}^{i}$ is given in terms of the Ricci tensor $R_{i j}$ by

$$
R_{j}^{i}=R_{h j}^{i h}=g^{i h} R_{h j} .
$$

A Schrödinger operator (or Hamiltonian) on $M$ is a second-order differential operator $h: \wedge^{0}(M) \rightarrow \wedge^{0}(M)$ of the form

$$
h=-\Delta+V,
$$


where $\Delta$ is the classical Laplace-Beltrami operator (7), and the potential $V$ is a scalar function. Just as the classical Laplace-Beltrami operator is the restriction of $-(d \delta+\delta d)$ to $\bigwedge^{0}(M)$, an arbitrary Schrödinger operator $h$ can be expressed in terms of a "twisted" version of the latter operator.

To this end, given a smooth real-valued function $\chi$ on $M$ we introduce the twisted differentials

$$
d^{ \pm}=e^{ \pm \chi} d e^{\mp \chi}
$$

and the twisted codifferentials

$$
\delta^{ \pm}=e^{ \pm \chi} \delta e^{\mp \chi}
$$

The operators $d^{ \pm}$and $\delta^{ \pm}$have the following properties, which follow directly from analogous properties of $d$ and $\delta$ :

$$
\begin{aligned}
\text { i) } & \left(d^{ \pm}\right)^{\dagger}=\delta^{\mp} \\
\text { ii) } & \left(d^{ \pm}\right)^{2}=\left(\delta^{ \pm}\right)^{2}=0 \\
\text { iii) } & d^{ \pm} \text {and } \delta^{ \pm} \text {are locally exact }
\end{aligned}
$$

There are two natural ways of "twisting" the operator $\Delta$ in such a way that the resulting operator maps $\wedge^{k}(M)$ into itself for $k=0,1, \ldots, n$, and is formally self-adjoint and nonnegative; namely, we can define the twisted Laplacians ${ }^{\dagger}$

$$
H=d^{-} \delta^{+}+\delta^{+} d^{-}
$$

and

$$
\widetilde{H}=d^{+} \delta^{-}+\delta^{-} d^{+} \text {. }
$$

Let us take the first of these operators, and apply it to a function $f \in \Lambda^{0}(M)$. We shall need the following lemma, whose proof is straightforward, to simplify the computation:

Lemma 1. If $f$ and $g$ are scalar functions on $M$, we have the identity

$$
\delta(f d g)=-f \Delta g-\nabla f \cdot \nabla g .
$$

Here $\nabla f$ denotes the gradient of $f$, that is the vector field with components

$$
(\nabla f)^{i}=g^{i j} \nabla_{j} f=g^{i j} \frac{\partial f}{\partial x^{j}},
$$

and $\nabla f \cdot \nabla g=\langle d f, d g\rangle$. Using the lemma we obtain the following expression for the action of the operator $H$ on a function $f \in \Lambda^{0}(M)$ :

$$
\begin{aligned}
H f & =\delta^{+} d^{-} f=e^{\chi} \delta\left[e^{-\chi} \cdot e^{-\chi} d\left(e^{\chi} f\right)\right] \\
& =e^{\chi} \delta\left(e^{-\chi} d f\right)+e^{\chi} \delta\left(e^{-\chi} f d \chi\right) \\
& =-\Delta f-e^{\chi} \nabla\left(e^{-\chi}\right) \cdot \nabla f-f \Delta \chi-e^{\chi} \nabla\left(e^{-\chi} f\right) \cdot \nabla \chi \\
& =\left(-\Delta+V-E_{0}\right) f,
\end{aligned}
$$

$\dagger$ The one-parameter family of twisted Laplacians $H_{t}$ obtained from $H$ by setting $\chi=$ $t \chi_{0}$, where $t \in \mathbb{R}$ and $\chi_{0}$ is a Morse function on $M$, was studied by Witten in his derivation of the Morse inequalities using supersymmetric quantum mechanics, cf. [17]. 
where

$$
V=(\nabla \chi)^{2}-\Delta \chi+E_{0}
$$

and $E_{0}$ is an arbitrary real constant whose purpose will soon become clear. (Note that $V$ is real, since $\chi$ is a real-valued function.) Thus, an arbitrary Schrödinger operator $h$ can be represented as

$$
h=H_{0}+E_{0} \equiv\left(\delta^{+} d^{-}+d^{-} \delta^{+}\right)_{0}+E_{0},
$$

provided that the function $\chi$ satisfies (10). The meaning of (10) and of the arbitrary constant $E_{0}$ is apparent if we observe that

$$
\left(h-E_{0}\right) e^{-\chi}=H_{0} e^{-\chi}=\delta^{+} d^{-} e^{-\chi}=\delta^{+}\left[e^{-\chi} d(1)\right]=0 .
$$

In other words, (10) is equivalent to the fact that $e^{-\chi}$ is a formal eigenfunction of $h$ with eigenvalue $E_{0}$. Note that here, and in what follows, by a formal eigenfunction of an operator $h$ we simply mean a solution $\psi$ of the eigenvalue equation $(h-E) \psi=0$, regardless of the boundary conditions (like square integrability) that may be used to define true eigenfunctions of $h$.

Similarly, if we apply the twisted Laplacian $\widetilde{H}$ defined above to a scalar function $f \in \Lambda^{0}(M)$ we obtain

$$
\widetilde{H} f=\delta^{-} d^{+} f=\left(-\Delta+\widetilde{V}-E_{0}\right) f
$$

with $\widetilde{V}$ given by

$$
\widetilde{V}=(\nabla \chi)^{2}+\Delta \chi+E_{0}=V+2 \Delta \chi
$$

since $\widetilde{H}$ is obtained from $H$ by replacing $\chi$ with $-\chi$. Letting

$$
\widetilde{h}=-\Delta+\widetilde{V}
$$

we then have

$$
\left(\widetilde{h}-E_{0}\right) e^{\chi}=0 .
$$

Note that the Schrödinger operator $\widetilde{h}$ is the Moutard transform of $h,[\mathbf{1 2}]$.

\section{The Multidimensional Darboux Transformation}

The goal of this section is to define the multidimensional Darboux transformation. The definition will be naturally suggested by some fundamental intertwining relations involving the differential operators on $\Lambda(M)$ introduced in the previous section.

Let us consider the operator $H: \wedge(M) \rightarrow \wedge(M)$, which we shall decompose as follows:

$$
H=H^{(1)}+H^{(2)}
$$

where

$$
H^{(1)}=d^{-} \delta^{+}, \quad H^{(2)}=\delta^{+} d^{-} .
$$


Observe that, since $\delta^{+}$and $d^{-}$are coboundary operators, we have

$$
H^{(1)} H^{(2)}=H^{(2)} H^{(1)}=0 .
$$

Since the operator $H$ maps $\wedge^{k}(M)$ into itself for $k=0,1, \ldots, n$, we can also write

$$
H=\bigoplus_{k=0}^{n} H_{k}
$$

where $H_{k}$ is the restriction of $H$ to $\wedge^{k}(M)$. The operator $H_{k}$ can be decomposed as

$$
H_{k}=H_{k}^{(1)}+H_{k}^{(2)}
$$

where $H_{k}^{(1)}$ and $H_{k}^{(2)}$ are the restrictions of $H^{(1)}$ and $H^{(2)}$ to $\wedge^{k}(M)$, namely

$$
H_{k}^{(1)}=d_{k-1}^{-} \delta_{k}^{+}, \quad H_{k}^{(2)}=\delta_{k+1}^{+} d_{k}^{-} .
$$

Notice that

$$
H_{0}^{(1)}=H_{n}^{(2)}=0 .
$$

From (13) we obtain

$$
H_{k}^{(1)} H_{k}^{(2)}=H_{k}^{(2)} H_{k}^{(1)}=0 .
$$

By construction, $H$ is formally self-adjoint and non-negative. Therefore the same is true for the operators $H_{k}: \wedge^{k}(M) \rightarrow \wedge^{k}(M)$ for $k=0,1, \ldots, n$. Likewise, both $H_{k}^{(1)}$ and $H_{k}^{(2)}$ are formally self-adjoint and non-negative, the latter property being a consequence of the identities

$$
\begin{aligned}
& \left(\alpha, H_{k}^{(1)} \alpha\right)=\left(d^{-} \alpha, d^{-} \alpha\right), \\
& \left(\alpha, H_{k}^{(2)} \alpha\right)=\left(\delta^{+} \alpha, \delta^{+} \alpha\right), \quad \alpha \in \bigwedge^{k}(M), \quad k=0,1, \ldots, n .
\end{aligned}
$$

The following intertwining relations are an immediate consequence of the definition of $H^{(1)}$ and $H^{(2)}$ :

$$
\begin{aligned}
& \delta^{+} H^{(1)}=H^{(2)} \delta^{+} \\
& H^{(1)} d^{-}=d^{-} H^{(2)} .
\end{aligned}
$$

These imply

$$
\begin{aligned}
\delta_{k+1}^{+} H_{k+1}^{(1)} & =H_{k}^{(2)} \delta_{k+1}^{+} \\
H_{k+1}^{(1)} d_{k}^{-} & =d_{k}^{-} H_{k}^{(2)}, \quad k=0,1, \ldots, n-1 .
\end{aligned}
$$

The intertwining relations (15)-(16) have important consequences for the spectra of the operators $H_{k+1}^{(1)}$ and $H_{k}^{(2)}$, that we shall now explore. 


\section{Proposition 2.}

i) If $\omega \in \Lambda^{k+1}(M)$ is an eigenform of $H_{k+1}^{(1)}$ with eigenvalue $\lambda \neq 0$, then $\delta^{+} \omega \equiv \delta_{k+1}^{+} \omega$ is an eigenform of $H_{k}^{(2)}$ with the same eigenvalue.

ii) Likewise, if $\omega \in \wedge^{k}(M)$ is an eigenform of $H_{k}^{(2)}$ with eigenvalue $\lambda \neq 0$, then $d^{-} \omega \equiv d_{k}^{-} \omega$ is an eigenform of $H_{k+1}^{(1)}$ with the same eigenvalue.

ProOF: The only point that is not an immediate consequence of the intertwining relations is that $\delta^{+} \omega$ in part i) cannot vanish identically, and likewise for $d^{-} \omega$ in part ii). Let us show, for instance, that $\delta^{+} \omega \neq 0$ in part i). If $\delta^{+} \omega=0$, then by the local exactness of $\delta^{+}$ (property iii) in Section 2) for every $p \in M$ there is an open neighborhood $U_{p}$ of $p$ such that $\omega=\delta^{+} \alpha_{p}$ on $U_{p}$, for some differential form $\alpha_{p}$ on $U_{p}$. But this would imply that $H_{k+1}^{(1)} \omega=d^{-} \delta^{+}\left(\delta^{+} \alpha_{p}\right)=0$ on $U_{p}$, for every $p \in M$, so that $H_{k+1}^{(1)} \omega=0$ on $M$. Hence $\lambda=0$, contradicting the hypothesis.

Q.E.D.

Let $L$ be a differential operator on $\Lambda(M)$ mapping each subspace $\Lambda^{k}(M)$ into itself. Examples of such an operator are $H, H^{(1)}$ and $H^{(2)}$. We shall denote by $\mathcal{A}(L) \subset \wedge(M)$ the set of admissible forms for $L$, that is the subspace of the space of square-integrable forms $L^{2}(\Lambda(M))$ satisfying any additional boundary or asymptotic conditions that are appropriate for the problem being considered. The set of admissible forms for $L_{k}$ is then $\mathcal{A}\left(L_{k}\right) \equiv \mathcal{A}_{k}(L)=\mathcal{A}(L) \cap \wedge^{k}(M)$. The spectrum of $L$, denoted by $\sigma(L)$, is the set of numbers $\lambda$ such that there is an admissible non-zero eigenform $\omega \in \mathcal{A}(L)$ satisfying the eigenvalue equation $L \omega=\lambda \omega$. We shall also use the convenient notation

$$
\sigma^{\prime}(L)=\sigma(L)-\{0\}
$$

The linear space of all $k$-forms $\omega$ satisfying the eigenvalue equation $L \omega=\lambda \omega$ will be denoted by $\Lambda_{\lambda}^{k}(L)$. Equivalently, $\Lambda_{\lambda}^{k}(L)$ is the set of eigenforms of $L_{k}$ with eigenvalue $\lambda$, together with the zero $k$-form.

By the formal self-adjointness and non-negativity of $H_{k}, H_{k}^{(1)}$ and $H_{k}^{(2)}$, the eigenvalues of these operators are real and non-negative. Proposition 2 has the following immediate corollary:

Corollary 3. If the operators $\delta^{+}$and $d^{-}$map $\mathcal{A} \equiv \mathcal{A}(H)$ into itself, then the spectra of $H_{k}^{(2)}$ and $H_{k+1}^{(1)}$ are related by

$$
\sigma^{\prime}\left(H_{k}^{(2)}\right)=\sigma^{\prime}\left(H_{k+1}^{(1)}\right), \quad k=0,1, \ldots, n-1 .
$$

In the remainder of this section, we shall not distinguish between true and formal eigenforms, unless otherwise indicated. The following lemma, whose proof is elementary, will have non-trivial consequences in what follows: 
Lemma 4. Let $V$ be a vector space, and let $L: V \rightarrow V$ be a linear operator. Suppose that $L=L_{1}+L_{2}$, with $L_{1} L_{2}=L_{2} L_{1}=0$. The following statements are then true:

i) If $v$ is an eigenvector of $L$ with eigenvalue $\lambda$, either $L_{i} v=\lambda v$ and $L_{j} v=0$ for some $i, j \in\{1,2\}$ with $i \neq j$, or $L_{i} v$ is an eigenvector of $L_{i}$ with eigenvalue $\lambda$ for $i=1,2$.

ii) $\sigma(L) \subset \sigma\left(L_{1}\right) \cup \sigma\left(L_{2}\right)$.

iii) $\sigma^{\prime}(L)=\sigma^{\prime}\left(L_{1}\right) \cup \sigma^{\prime}\left(L_{2}\right)$.

From the previous lemma and (14), it follows that

$$
\sigma^{\prime}\left(H_{k}\right)=\sigma^{\prime}\left(H_{k}^{(1)}\right) \cup \sigma^{\prime}\left(H_{k}^{(2)}\right)
$$

Thus, the spectrum of $H_{k}$, with the possible exception of the zero eigenvalue, is simply the union of the spectra of its components $H_{k}^{(1)}$ and $H_{k}^{(2)}$. The spectra of $H_{k+1}^{(1)}$ and $H_{k}^{(2)}$ $(k=0,1, \ldots, n-1)$ are identical, except perhaps for the zero eigenvalue. The operator $\delta^{+}$maps eigenforms of $H_{k+1}^{(1)}$ with non-zero eigenvalue into eigenforms of $H_{k}^{(2)}$ with the same eigenvalue, and similarly $d^{-}$maps eigenforms of $H_{k}^{(2)}$ with non-zero eigenvalue into eigenforms of $H_{k+1}^{(1)}$ with the same eigenvalue. From the identity (17) we easily obtain the following relation between the even and odd components of $H$ :

$$
\sigma^{\prime}\left(\bigoplus_{j=0}^{[n / 2]} H_{2 j}\right)=\sigma^{\prime}\left(\bigoplus_{j=0}^{\left[\frac{n-1}{2}\right]} H_{2 j+1}\right)
$$

Definition 5. Let $\lambda \neq 0$, and $k=0,1, \ldots, n-1$. If $\omega \in \Lambda_{\lambda}^{k+1}\left(H^{(1)}\right)$, its Darboux transform is the $k$-form $\delta^{+} \omega \in \Lambda_{\lambda}^{k}\left(H^{(2)}\right)$. Similarly, the Darboux transform of a $k$-form $\omega \in \Lambda_{\lambda}^{k}\left(H^{(2)}\right)$ is the $(k+1)$-form $d^{-} \omega \in \Lambda_{\lambda}^{k+1}\left(H^{(1)}\right)$.

From the definition of $H^{(i)}$ it directly follows that if $\lambda \neq 0$ the restriction $\delta^{+}$: $\bigwedge_{\lambda}^{k+1}\left(H^{(1)}\right) \rightarrow \bigwedge_{\lambda}^{k}\left(H^{(2)}\right)$ is invertible, its inverse being the restriction $\lambda^{-1} d^{-}: \bigwedge_{\lambda}^{k}\left(H^{(2)}\right) \rightarrow$ $\Lambda_{\lambda}^{k+1}\left(H^{(1)}\right)$.

The Darboux transformation we have just defined acts in a natural way on eigenforms of the partial Hamiltonians $H^{(1)}$ and $H^{(2)}$. In the same spirit, we shall see next how to use the Darboux transformation to construct new eigenforms of $H$ of degree $k-1$ and/or $k+1$ starting from a given eigenform of degree $k$ with non-zero eigenvalue. To this end, let $\omega \in \Lambda^{k}(M)$ be an eigenform of $H$ with eigenvalue $\lambda \neq 0$. By Lemma 4 , either $H^{(i)} \omega=\lambda \omega$ for some $i \in\{1,2\}$, or $H^{(i)} \omega$ is an eigenform of $H^{(i)}$ with eigenvalue $\lambda \neq 0$ for $i=1,2$. In the first case, the Darboux transform of $\omega$ is well defined according to Definition 5, and belongs to either $\Lambda_{\lambda}^{k-1}\left(H^{(2)}\right)$ (when $i=1$ ), or to $\Lambda_{\lambda}^{k+1}\left(H^{(1)}\right)$ (when $i=2$ ). By Lemma 4 , it follows that the Darboux transform of $\omega$ is an eigenform of $H$ in this case. In the second case, the Darboux transforms of both $H^{(1)} \omega$ and $H^{(2)} \omega$ are defined and, as before, are eigenforms of $H$ with eigenvalue $\lambda$. Hence in this case both $\delta^{+} H^{(1)} \omega \equiv \delta^{+} d^{-} \delta^{+} \omega$ and $d^{-} H^{(2)} \omega \equiv d^{-} \delta^{+} d^{-} \omega$ are eigenforms of $H$ with eigenvalue $\lambda$ and degree equal to $k-1$ 
and $k+1$, respectively. Note that in this case both $H^{(1)} \omega$ and $H^{(2)} \omega$ are also eigenforms of $H$ of degree $k$ with eigenvalue $\lambda$, neither of which is proportional to $\omega$ (although the span of $\omega, H^{(1)} \omega$ and $H^{(2)} \omega$ is obviously two-dimensional). Thus in the second case, i.e, when $H^{(i)} \omega \neq 0$ for $i=1,2$, we can construct three new eigenforms of $H$ of degrees $k-1$, $k$ and $k+1$ and eigenvalue $\lambda \neq 0$ starting from a known eigenform $\omega \in \wedge_{\lambda}^{k}(H)$.

The above construction could have been carried out using the twisted Laplacian $\widetilde{H}$ instead of $H$. However, we shall now show that the two constructions are equivalent:

Proposition 6. For $k=0,1, \ldots, n$, the operators $H_{n-k}$ and $\widetilde{H}_{k}$ are linearly equivalent under Hodge duality:

$$
\widetilde{H}_{k}=(*)^{-1} H_{n-k} *
$$

Proof: An elementary calculation shows that

$$
* H_{n-k} *=(-1)^{k(n-k)} \widetilde{H}_{k}
$$

from which (19) follows using (3).

Q.E.D.

In other words, if we identify the space $\wedge^{n-k}(M)$ to $\wedge^{k}(M)$ under the Hodge star operator, $H_{n-k}$ is transformed into $\widetilde{H}_{k}$. In particular, $H_{n}$ is equivalent under this identification to $\widetilde{H}_{0}=\widetilde{h}-E_{0}$, the Moutard transform of $H_{0}=h-E_{0}$.

We end this section by deriving local coordinate expressions for the multidimensional Darboux transformation and for the component Hamiltonians $H_{k}$ and $\widetilde{H}_{k}$. By definition,

$$
d^{-} \omega=e^{-\chi} d\left(e^{\chi} \omega\right)=d \omega+d \chi \wedge \omega
$$

so that if $\omega \in \Lambda^{k}(M)$ we have the following local coordinate expression

$$
\begin{aligned}
\left(d^{-} \omega\right)_{j i_{1} \ldots i_{k}} & =\left(\nabla_{[j}+\chi_{j}\right) \omega_{\left.i_{1} \ldots i_{k}\right]} \\
& =\left(\nabla_{j}+\chi_{j}\right) \omega_{i_{1} \ldots i_{k}}-\sum_{r=1}^{k}\left(\nabla_{i_{r}}+\chi_{i_{r}}\right) \omega_{i_{1} \ldots i_{r-1} j i_{r+1} \ldots i_{k}}
\end{aligned}
$$

Here the square brackets denote antisymmetrization, and $\chi_{j}=\nabla_{j} \chi=\partial \chi / \partial x^{j}$. Similarly, using (6) we obtain

$$
\left(\delta^{+} \omega\right)_{i_{1} \ldots i_{k-1}}=\left(-\nabla^{j}+\chi^{j}\right) \omega_{j i_{1} \ldots i_{k-1}}, \quad \omega \in \bigwedge^{k}(M)
$$

Using the local formulas (20) and (21) we obtain, after a straightforward calculation

$$
\begin{aligned}
\left(H_{k} \omega\right)_{i_{1} \ldots i_{k}} & =\left[-\nabla_{j} \nabla^{j}+(\nabla \chi)^{2}-\Delta \chi\right] \omega_{i_{1} \ldots i_{k}} \\
& +\sum_{r=1}^{k}\left\{\nabla^{j}, \nabla_{i_{r}}\right\} \chi \cdot \omega_{i_{1} \ldots i_{r-1} j i_{r+1} \ldots i_{k}}
\end{aligned}
$$




$$
+\sum_{r=1}^{k}\left[\nabla^{j}, \nabla_{i_{r}}\right] \omega_{i_{1} \ldots i_{r-1} j i_{r+1} \ldots i_{k}}
$$

This formula can be further simplified as follows. In the first place, note that since $\chi$ is a scalar function we have

$$
\left\{\nabla^{j}, \nabla_{i_{r}}\right\} \chi=2 \nabla^{j} \nabla_{i_{r}} \chi=2 \nabla_{i_{r}} \nabla^{j} \chi
$$

Secondly, from the identity

$$
\left[\nabla_{j}, \nabla_{l}\right] \omega_{i_{1} \ldots i_{k}}=\sum_{r=1}^{k} R_{i_{r} j l}^{h} \omega_{i_{1} \ldots i_{r-1} h i_{r+1} \ldots i_{k}}
$$

we obtain, after straightforward manipulations

$$
\begin{aligned}
\sum_{r=1}^{k}\left[\nabla^{j}, \nabla_{i_{r}}\right] \omega_{i_{1} \ldots i_{r-1} j i_{r+1} \ldots i_{k}} & =\sum_{r=1}^{k}(-1)^{r+1} R_{i_{r}}^{h} \omega_{h i_{1} \ldots \widehat{i_{r} \ldots i_{k}}} \\
& +\sum_{1 \leq r<q \leq k}(-1)^{r+q+1} R_{i_{r}{ }_{i_{q}}{ }^{h} \omega_{j h i_{1} \ldots \widehat{i_{r} \ldots} \ldots \widehat{i_{q} \ldots i_{k}}}} .
\end{aligned}
$$

Substituting the previous formulas into (22) we get the following coordinate expression for the action of $H_{k}$ :

$$
\begin{aligned}
\left(H_{k} \omega\right)_{i_{1} \ldots i_{k}} & =\left[-\nabla_{j} \nabla^{j}+(\nabla \chi)^{2}-\Delta \chi\right] \omega_{i_{1} \ldots i_{k}} \\
& +2 \sum_{r=1}^{k} \nabla^{j} \nabla_{i_{r}} \chi \cdot \omega_{i_{1} \ldots i_{r-1}} j i_{r+1} \ldots i_{k} \\
& +\sum_{r=1}^{k}(-1)^{r+1} R^{h}{ }_{i_{r}} \omega_{h i_{1} \ldots \widehat{i_{r} \ldots i_{k}}} \\
& +\sum_{1 \leq r<q \leq k}(-1)^{r+q+1} R_{i_{r}{ }^{j}{ }_{i_{q}}} \omega_{j h i_{1} \ldots \hat{i}_{r} \ldots \hat{i_{q}} \ldots i_{k}} .
\end{aligned}
$$

When $\chi=0$ the previous expression reduces to the formula for minus the Laplacian, eq. (8). In particular, the operator $H_{k}$ has the structure

$$
H_{k}=-\Delta_{k}+V_{k}
$$

where $\Delta_{k}$ is the restriction of the Laplacian to $\Lambda^{k}(M)$, and $V_{k}$ acts like a matrix potential on the components of any $k$-form:

$$
\left(V_{k} \omega\right)_{i_{1} \ldots i_{k}}=\left[(\nabla \chi)^{2}-\Delta \chi\right] \omega_{i_{1} \ldots i_{k}}+2 \sum_{r=1}^{k} \nabla^{j} \nabla_{i_{r}} \chi \cdot \omega_{i_{1} \ldots i_{r-1} j i_{r+1} \ldots i_{k}}
$$




$$
=\left(V-E_{0}\right) \omega_{i_{1} \ldots i_{k}}+2 \sum_{r=1}^{k} \nabla^{j} \nabla_{i_{r}} \chi \cdot \omega_{i_{1} \ldots i_{r-1} j i_{r+1} \ldots i_{k}} .
$$

The local coordinate expressions for the component Hamiltonians $\widetilde{H}_{k}$ are obtained from the previous formulas by replacing $\chi$ with $-\chi$.

As first pointed out by Witten, $[\mathbf{1 6}],[\mathbf{1 7}],[\mathbf{2}]$, the connection between the spectra of the component Hamiltonians $H_{k}$ discussed in this section admits an interesting interpretation in terms of supersymmetry. Indeed, the $n+1$ homogeneous components $\omega_{k}$ of degree $k$ of a differential form $\Omega=\bigoplus_{k=0}^{n} \omega_{k}$ can be interpreted as the components of a supermultiplet, with $k$-forms regarded as being bosonic or fermionic depending on whether $k$ is even or odd, respectively. The supercharges $Q^{ \pm}$are by definition the operators

$$
Q^{-}=\delta^{+}, \quad Q^{+}=\left(Q^{-}\right)^{\dagger}=d^{-},
$$

while the supersymmetric Hamiltonian

$$
H=\left\{Q^{+}, Q^{-}\right\}
$$

is just the twisted Laplacian. The remaining commutation relations defining the standard supersymmetry algebra

$$
\left\{Q^{ \pm}, Q^{ \pm}\right\}=\left[Q^{ \pm}, H\right]=0
$$

hold thanks to the elementary properties of $d^{ \pm}, \delta^{ \pm}$.

We can also introduce fermion creation and annihilation operators

$$
\left.b_{i}^{-}=\frac{\partial}{\partial x^{i}}\right\lrcorner, \quad b^{i+}=d x^{i} \wedge
$$

where $\downarrow$ denotes the inner product. The usual fermionic anticommutation relations

$$
\left\{b^{i+}, b^{j+}\right\}=\left\{b_{i}^{-}, b_{j}^{-}\right\}=0, \quad\left\{b^{i+}, b_{j}^{-}\right\}=\delta_{j}^{i},
$$

as well as the identity

$$
b^{i+}=\left(b^{i-}\right)^{\dagger} \equiv\left(g^{i j} b_{j}^{-}\right)^{\dagger},
$$

follow easily from well known exterior algebra identities. The supercharges $Q^{ \pm}$can be expressed in terms of the creation and annihilation operators as

$$
Q^{ \pm}=q^{i \mp} b_{i}^{ \pm}
$$

where

$$
q_{i}^{ \pm} \omega=\frac{1}{k !}\left(\mp \nabla_{i}+\nabla_{i} \chi\right) \omega_{i_{1} \ldots i_{k}} \cdot d x^{i_{1}} \wedge \ldots \wedge d x^{i_{k}}=g_{i j} q^{j \pm} \omega .
$$

One can easily check the identity

$$
H=-\Delta+(\nabla \chi)^{2}+\nabla_{i} \nabla_{j} \chi \cdot\left[b^{i+}, b^{j-}\right],
$$

which generalizes formula (13) of [17] (where an orthonormal basis of the tangent space is used to define creation and annihilation operators). 


\section{The Darboux Transformation in Low Dimensions}

Let us begin by showing that in the one-dimensional case the multidimensional Darboux transformation reduces to the classical Darboux transformation. Indeed, in this case $M=$ $\mathbb{R}, H_{0}=h-E_{0}$, and $H_{1}$ is equivalent to $\widetilde{H}_{0}=\widetilde{h}-E_{0}$ under the Hodge duality. If

$$
h=-\frac{d^{2}}{d x^{2}}+V(x)
$$

then from (11) we have

$$
\widetilde{h}=-\frac{d^{2}}{d x^{2}}+\widetilde{V}(x)
$$

where

$$
\widetilde{V}=V+2 \chi^{\prime \prime}
$$

and

$$
\left(h-E_{0}\right) e^{-\chi}=0 .
$$

The operator $d^{-}$maps eigenfunctions of $h=H_{0}+E_{0}$ with eigenvalue $E \neq E_{0}$ into eigenfunctions of $H_{1}$ with eigenvalue $E-E_{0}$. Therefore, the operator $Q^{-}=* d^{-}$will map eigenfunctions of $h$ with eigenvalue $E \neq E_{0}$ into eigenfunctions of $\widetilde{h}$ with the same eigenvalue. If $x$ is a cartesian coordinate we easily obtain

$$
Q^{-} \psi=* d^{-} \psi=\left(\frac{d}{d x}+\chi^{\prime}\right) \psi
$$

so that

$$
Q^{-}=\frac{d}{d x}+\chi^{\prime}
$$

Similarly, if $\phi$ is an eigenfunction of $\widetilde{h}=\widetilde{H}+E_{0}$ with eigenvalue $E$ then $* \phi$ will be an eigenfunction of $H_{1}$ with eigenvalue $E-E_{0}$, so that $\delta^{+}(* \phi)$ is an eigenfunction of $h$ with eigenvalue $E$. Proceeding as before we have

$$
\delta^{+}(* \phi)=\left(-\frac{d}{d x}+\chi^{\prime}\right) \phi
$$

so that the operator

$$
Q^{+}=-\frac{d}{d x}+\chi^{\prime}
$$

maps eigenfunctions of $\widetilde{h}$ with eigenvalue $E \neq E_{0}$ into eigenfunctions of $h$ with the same eigenvalue. From the properties of $d^{ \pm}$and $\delta^{ \pm}$, it follows that $Q^{-}$and $Q^{+}$are formally the adjoint of one another under the Euclidean scalar product on $\mathbb{R}$, and that

$$
h-E_{0}=Q^{+} Q^{-}, \quad \widetilde{h}-E_{0}=Q^{-} Q^{+} .
$$

Equations (24), (25), (26) and (27) express the classical Darboux transformation. 
The two-dimensional Darboux transformation generalizes to curved oriented surfaces the Darboux transformation introduced in $[\mathbf{1}]$ for $\mathbb{R}^{2}$ in cartesian coordinates. Indeed, let $M$ be a two-dimensional oriented Riemannian manifold. The component Hamiltonians are in this case $H_{0}=h-E_{0}, H_{1}: \Lambda^{1}(M) \rightarrow \Lambda^{1}(M)$ and $H_{2}: \Lambda^{2}(M) \rightarrow \Lambda^{2}(M)$, which is equivalent under the Hodge duality to $\widetilde{H}_{0}=\widetilde{h}-E_{0}$. The scalar Hamiltonians $h$ and $\widetilde{h}$ are given by eqs. (9), (10), (12) and (11), while the function $e^{-\chi}$ as usual satisfies $\left(h-E_{0}\right) e^{-\chi}=0$. To the operator $H_{1}$ acting on one-forms there corresponds an operator $\hat{H}_{1}$ acting on vector fields, defined by

$$
\hat{H}_{1} X=\left(H_{1} X^{b}\right)^{\sharp},
$$

where $X^{b}=g_{i j} X^{j} d x^{i}$ is the one-form associated to the vector field $X=X^{i} \partial / \partial x^{i}$, and $\sharp=b^{-1}$. Using (23) we easily find the following local coordinate expression for $\hat{H}_{1} X$ :

$$
\left(\hat{H}_{1} X\right)^{i}=\left[-\nabla_{j} \nabla^{j}+(\nabla \chi)^{2}-\Delta \chi\right] X^{i}+2 \nabla^{i} \nabla_{j} \chi \cdot X^{j}+R_{j}^{i} X^{j} .
$$

From the well-known two-dimensional identity

$$
R_{j}^{i}=K \delta_{j}^{i}
$$

where $K$ is the Gaussian curvature of $M$, we obtain the equivalent expression

$$
\left(\hat{H}_{1} X\right)^{i}=\left[-\nabla_{j} \nabla^{j}+(\nabla \chi)^{2}-\Delta \chi+K\right] X^{i}+2 \nabla^{i} \nabla_{j} \chi \cdot X^{j} .
$$

In flat space and cartesian coordinates, the above formula for $\hat{H}_{1}$ reduces to formula (9) of [1]. By Corollary 3 and eq. (18), the spectra of the Hamiltonians $h, \widetilde{h}$ and $\hat{H}_{1}$ are related by

$$
\sigma^{\prime}\left(\hat{H}_{1}\right)=\sigma^{\prime}\left(h-E_{0}\right) \cup \sigma^{\prime}\left(\widetilde{h}-E_{0}\right) .
$$

We shall now derive an expression for the two-dimensional Darboux transformation in local coordinates. Let the operators $q_{i}^{ \pm}$and $p_{i}^{ \pm}$be defined in local coordinates by

$$
\begin{aligned}
& q_{i}^{ \pm}=\mp \nabla_{i}+\chi_{i}=\mp e^{ \pm \chi} \nabla_{i} e^{\mp \chi} \\
& p_{i}^{ \pm}=\sqrt{g} \epsilon_{i j} q^{j \mp} .
\end{aligned}
$$

In particular, notice that the operators $q_{i}^{ \pm}$(resp. $p_{i}^{ \pm}$) transform like the components of a covariant tensor (resp. pseudo-tensor) of rank one under changes of local coordinates.

If $\psi$ is an eigenfunction of $h$ with eigenvalue $E \neq E_{0}$, then $d^{-} \psi$ is an eigenfunction of $H_{1}$ with eigenvalue $E-E_{0}$. Using the general formula (20) we have

$$
\left(d^{-} \psi\right)_{i}=q_{i}^{-} \psi
$$


From (28) it follows that the vector field with components

$$
g^{i j} q_{j}^{-} \psi \equiv q^{j-} \psi
$$

is an eigenvector of $\hat{H}_{1}$ with eigenvalue $E-E_{0}$. Suppose now that $\phi$ is an eigenfunction of $\widetilde{h}$ with eigenvalue $E \neq E_{0}$. Then $* \phi$ is an eigenform of $H_{2}$ with eigenvalue $E-E_{0}$, so that $\delta^{+}(* \phi)$ is an eigenform of $H_{1}$ with eigenvalue $E-E_{0}$. From the local formulas (5) and (21) and the fact that the pseudo-tensor with components $\sqrt{g} \epsilon_{i j}$ is covariantly constant, it follows that

$$
\delta^{+}(* \phi)_{i}=-p_{i}^{-} \phi
$$

Therefore the vector field with components

$$
g^{i j} p_{j}^{-} \phi \equiv p^{i-} \phi
$$

is an eigenvector of $\hat{H}_{1}$ with eigenvalue $E-E_{0}$. Note also that $p^{i \pm}$ can be expressed as

$$
p^{i \pm}=\frac{1}{\sqrt{g}} \sum_{j} \epsilon_{i j} q_{j}^{\mp}
$$

Conversely, if $\Psi=\psi_{i} d x^{i}$ is an eigenform of $H_{1}^{(1)}$ with eigenvalue $\lambda \neq 0$, then (Lemma 4) $\Psi$ is an eigenform of $H_{1}$ with the same eigenvalue, and $\delta^{+} \Psi$ is an eigenfunction of $h$ with eigenvalue $\lambda+E_{0}$. From (6) we obtain

$$
\delta^{+} \Psi=-e^{\chi} \nabla^{i}\left(e^{-\chi} \psi_{i}\right)=q^{i+} \psi_{i}=q_{i}^{+} \psi^{i},
$$

where the vector field $\Psi^{\sharp}=\psi^{i} \partial / \partial x^{i}$ is an eigenvector of $\hat{H}_{1}$. Likewise, if $\Psi$ is an eigenform of $H_{1}^{(2)}$ with eigenvalue $\lambda \neq 0$ then $\Psi$ is an eigenform of $H_{1}$ with the same eigenvalue, and $\delta^{+} \Psi$ is an eigenfunction of $H_{2}$ with eigenvalue $\lambda+E_{0}$. It follows that $* \delta^{+} \Psi$ is an eigenfunction of $\widetilde{h}$ with eigenvalue $\lambda+E_{0}$. Using the local coordinate formulas (5) and (20) we easily obtain

$$
* d^{-} \Psi=p_{i}^{+} \psi^{i},
$$

where again the functions $\psi^{i}$ are the components of an eigenvector of $\hat{H}_{1}$ with eigenvalue $\lambda$. As before, in flat space and cartesian coordinates eqs. (30), (32)-(35) reduce to the corresponding formulas in [1].

The above formulas expressing the two-dimensional Darboux transformation in terms of the operators $q_{i}^{ \pm}$and $p_{i}^{ \pm}$suggest that the component Hamiltonians and the intertwining relations can also be written in terms of the latter operators. A straightforward computation using the local coordinate expressions for $*, d$ and $\delta$ shows that this is indeed the case. More precisely, we have

$$
h-E_{0}=q_{i}^{+} q^{i-}, \quad \widetilde{h}-E_{0}=q_{i}^{-} q^{i+}=p_{i}^{+} p^{i-}
$$


and $\hat{H}_{1}=\hat{H}_{1}^{(1)}+\hat{H}_{1}^{(2)}$, with

$$
\left(\hat{H}_{1}^{(1)} \Psi\right)^{i}=q^{i-} q_{j}^{+} \psi^{j} \equiv\left(\hat{H}^{(1)}\right)_{j}^{i} \psi^{j}, \quad\left(\hat{H}_{1}^{(2)} \Psi\right)^{i}=p^{i-} p_{j}^{+} \psi^{j} \equiv\left(\hat{H}^{(2)}\right)_{j}^{i} \psi^{j} .
$$

Notice that, strictly speaking, $\left(\hat{H}^{(k)}\right)^{i}{ }_{j}$ is not the $(i, j)$-th matrix element of the operator $\hat{H}^{(k)}$, since $\nabla_{i} \psi^{j}$ in general depends on all the components of $\Psi$. Similarly, the intertwining relations (15)-(16) can be written as

$$
\begin{aligned}
q_{i}^{+}\left(\hat{H}^{(1)}\right)_{j}^{i} & =\left(h-E_{0}\right) q_{j}^{+} \\
\left(\hat{H}^{(1)}\right)_{j}^{i} q^{j-} & =q^{i-}\left(h-E_{0}\right) \\
p_{i}^{+}\left(\hat{H}^{(2)}\right)_{j}^{i} & =\left(\widetilde{h}-E_{0}\right) p_{j}^{+} \\
\left(\hat{H}^{(2)}\right)_{j}^{i} p^{j-} & =p^{i-}\left(\widetilde{h}-E_{0}\right) .
\end{aligned}
$$

In flat space and cartesian coordinates, the above expressions reduce to the corresponding ones in $[\mathbf{1}]$.

We shall now show how the classical Moutard transform, [12], is generalized in the case of an oriented Riemannian surface. To this end, suppose that $\psi$ is a formal eigenfunction of $h$ with the same eigenvalue $E_{0}$ as $e^{-\chi}$. Note that $\psi$ need not be proportional to $e^{-\chi}$, since we are dealing with formal eigenfunctions. A Moutard transform of $\psi$ is any function $\tilde{\psi}$ satisfying

$$
d^{+} \tilde{\psi}=\delta^{-}(* \psi)
$$

Locally, the above equation has a solution if and only if $\left(h-E_{0}\right) \psi=0$. Indeed, by the local exactness of $d^{+}$the compatibility condition of (36) is

$$
d^{+} \delta^{-}(* \psi)=0 \text {. }
$$

The Hodge dual of the latter equation is simply

$$
\delta^{+} d^{-} \psi=\left(h-E_{0}\right) \psi=0
$$

as claimed. Note that the Moutard transform $\tilde{\psi}$ is locally defined by (36) only up to a constant multiple of $e^{\chi}$. Indeed, if $\tilde{\psi}_{1}$ and $\tilde{\psi}_{2}$ are two Moutard transforms of $\psi$ then their difference satisfies $d^{+}\left(\tilde{\psi}_{1}-\tilde{\psi}_{2}\right)=0$, that is $d\left[e^{-\chi}\left(\tilde{\psi}_{1}-\tilde{\psi}_{2}\right)\right]=0$.

If $\tilde{\psi}$ is any Moutard transform of $\psi$, then

$$
\left(\widetilde{h}-E_{0}\right) \tilde{\psi}=0,
$$

where as before $\widetilde{h}$ is the Moutard transform of $h$. Indeed, applying $\delta^{-}$to (36) we obtain

$$
0=\delta^{-} d^{+}(\tilde{\psi})=\widetilde{H}_{0} \tilde{\psi}=\left(\widetilde{h}-E_{0}\right) \tilde{\psi}
$$




\section{The Multidimensional Moutard Transformation}

We shall derive in this section a generalization to oriented Riemannian manifolds of arbitrary dimension of the classical two-dimensional Moutard transformation introduced in the previous section. The key to this generalization is a remarkable connection between the zero eigenspaces of the operators $H_{k}^{(2)}$ and $H_{k+2}^{(1)}$ (or, equivalently, of the components $\widetilde{H}_{k}^{(2)}$ and $\widetilde{H}_{k-2}^{(1)}$ of $\widetilde{H}$ defined below) that we shall describe next.

Throughout this section, $M$ will denote an oriented Riemannian manifold of dimension $n \geq 2$ with trivial de Rham cohomology. In particular, the latter condition will always hold if we restrict ourselves to a contractible open subset of $M$. Let us decompose $\widetilde{H}$ as

$$
\widetilde{H}=\widetilde{H}^{(1)}+\widetilde{H}^{(2)}
$$

with

$$
\widetilde{H}^{(1)}=\delta^{-} d^{+}, \quad \widetilde{H}^{(2)}=d^{+} \delta^{-},
$$

so that

$$
\widetilde{H}^{(i)}=(*)^{-1} H^{(i)} *, \quad i=1,2,
$$

as in Proposition 6. As before, the operators $H^{(i)}$ are formally non-negative and are the formal adjoint of one another. If $k=2,3, \ldots, n$, let $\omega \in \Lambda_{0}^{k}\left(\widetilde{H}^{(2)}\right)$ be a zero mode of $\widetilde{H}^{(2)}$, i.e, a $k$-form $\omega$ satisfying the equation $\widetilde{H}^{(2)} \omega=0$. In this section we shall exclusively deal with formal eigenforms, so that in particular $\omega$ is not required to be square-integrable. By analogy with (36), it is natural to consider the equation

$$
d^{+} \bar{\omega}=\delta^{-} \omega
$$

as an equation for the $(k-2)$-form $\bar{\omega}$. The properties of $(37)$, which are easily established, are analogous to those of (36), namely:

i) Equation (37) is compatible if and only if $\omega$ is a zero mode of $\widetilde{H}^{(2)}$.

ii) $\bar{\omega}$ is uniquely defined by (37) up to an element of $\operatorname{Im} d_{k-3}^{+}=e^{\chi} \operatorname{Im} d_{k-3}$. In particular, if $k=2$ we have $\operatorname{Im} d_{-1}=\mathbb{R}$ (the space of constant functions on $M$ ), so that $\operatorname{Im} d_{-1}^{+}=$ $\mathbb{R} e^{\chi}$.

iii) Any solution $\bar{\omega}$ of $(37)$ is a zero mode of $\widetilde{H}_{k-2}^{(1)}$, i.e., we have

$$
\widetilde{H}^{(1)} \bar{\omega}=0
$$

From the above properties it follows that (37) defines a mapping

$$
-\bigwedge_{0}^{k}\left(\widetilde{H}^{(2)}\right) / \operatorname{Im} \delta_{k+1}^{-} \rightarrow \bigwedge_{0}^{k-2}\left(\widetilde{H}^{(1)}\right) / \operatorname{Im} d_{k-3}^{+}, \quad 2 \leq k \leq n
$$

which is easily seen to be an isomorphism by the assumption on the de Rham cohomology of $M$. Note that for the above mapping to be non-trivial $M$ must be non-compact. Indeed, if 
$M$ is compact then an easy integration by parts argument implies that $\bigwedge_{0}^{k}\left(\widetilde{H}^{(2)}\right)=\operatorname{Im} \delta_{k+1}^{-}$ and $\bigwedge_{0}^{k-2}\left(\widetilde{H}^{(1)}\right)=\operatorname{Im} d_{k-3}^{+}$.

Totally analogous considerations can be made for the components $H_{k}^{(2)}$ and $H_{k+2}^{(1)}$ of $H$. To be precise, consider the equation

$$
\delta^{+} \breve{\omega}=d^{-} \omega
$$

where $\omega$ is a $k$-form and $k=0,1, \ldots, n-2$. As before, the integrability condition for (38) is that $\omega$ be a zero mode of $H_{k}^{(2)}$, in which case $\breve{\omega} \in \Lambda^{k+2}(M)$ is uniquely defined modulo $\operatorname{Im} \delta_{k+3}^{+}=e^{\chi} \operatorname{Im} \delta_{k+3}\left(\right.$ with $\left.\operatorname{Im} \delta_{n+1}=*\left(\operatorname{Im} \delta_{-1}\right)=\mathbb{R} \mu\right)$, and is a zero mode of $H_{k+2}^{(1)}$. Equation (38) thus defines a mapping

$$
: \bigwedge_{0}^{k}\left(H^{(2)}\right) / \operatorname{Im} d_{k-1}^{-} \rightarrow \bigwedge_{0}^{k+2}\left(H^{(1)}\right) / \operatorname{Im} \delta_{k+3}^{+}, \quad 0 \leq k \leq n-2
$$

which is again an isomorphism. Clearly, the mappings - and ${ }^{`}$ are related by Hodge duality. More precisely, an elementary calculation yields the following result:

Proposition 7. The maps $\left({ }^{\cup}\right)_{k}$ and $-(-)_{n-k}$ are conjugated under Hodge duality:

$$
\left({ }^{u}\right)_{k}=-(*)^{-1} \circ(-)_{n-k} \circ * \text {. }
$$

Comparing equations (36) and (37), we see that a Moutard transform of a function $\psi \in \Lambda_{0}^{0}\left(H^{(2)}\right) \equiv \Lambda_{0}^{0}(H)$ on a two-dimensional manifold $M$ is simply any function $\tilde{\psi}$ in the equivalence class of $\overline{* \psi}$, which will be an element of $\bigwedge_{0}^{0}\left(\widetilde{H}^{(1)}\right) \equiv \bigwedge_{0}^{0}(\widetilde{H})$. This observation motivates the following general definition:

Definition 8. The Moutard transform of $\omega \in \Lambda_{0}^{k}\left(H^{(2)}\right) / \operatorname{Im} d_{k-1}^{-}$is the element

$$
\widetilde{\omega}=\overline{* \omega}
$$

of $\bigwedge_{0}^{n-k-2}\left(\widetilde{H}^{(1)}\right) / \operatorname{Im} d_{n-k-3}^{+}$.

The domain and the range of the Moutard operator $\sim$ defined above follow immediately from the identity

$$
\sim=-\circ *
$$

or equivalently, using (39),

$$
\sim=-\left(* 0^{\cup}\right) \text {. }
$$

In other words, if $\omega \in \bigwedge_{0}^{k}\left(H^{(2)}\right) / \operatorname{Im} d_{k-1}^{-}$its Moutard transform is the unique solution $\widetilde{\omega} \in \Lambda^{n-k-2}(M) / \operatorname{Im} d_{n-k-3}^{+}$of the equation

$$
d^{+} \widetilde{\omega}=\delta^{-}(* \omega)
$$

which is automatically an element of $\bigwedge_{0}^{n-k-2}\left(\widetilde{H}^{(1)}\right) / \operatorname{Im} d_{n-k-3}^{+}$. As before, for the Moutard operator to be non-trivial $M$ must be non-compact, since otherwise $\bigwedge_{0}^{k}\left(H^{(2)}\right)=$ $\operatorname{Im} d_{k-1}^{-}$and $\bigwedge_{0}^{n-k-2}\left(\widetilde{H}^{(1)}\right)=\operatorname{Im} d_{n-k-3}^{+}$. Notice also that the Moutard transform of a $k^{-}$ form $\omega$ has different degree than $\omega$, unless $n=2 m$ is even and $k=m-1$, with $m=1,2, \ldots$ For instance, when $m=1$ we have $n=2, k=n-k-2=0$, and we obtain the generalization of the classical Moutard transformation to Riemannian surfaces introduced in the previous section. 


\section{Examples}

We present in this section a few examples of the two-dimensional Darboux transformation on curved surfaces based on the theory of quasi-exactly solvable Hamiltonians, $[\mathbf{7}],[\mathbf{8}]$.

As our first example, consider the first-order differential operators

$$
J^{1}=\partial_{x}, \quad J^{2}=\partial_{y}, \quad J^{3}=x \partial_{x}, \quad J^{4}=x \partial_{y}, \quad J^{5}=y \partial_{y}, \quad J^{6}=x^{2} \partial_{x}+x y \partial_{y}-2 x .
$$

The above differential operators span a Lie algebra $\mathfrak{g} \simeq \mathfrak{g l}(2, \mathbb{R}) \ltimes \mathbb{R}^{2}$, which coincides with the canonical form 1.11 in the classification of Lie algebras of differential operators in two variables of reference [9] for $n=r=2$. The latter Lie algebra is quasi-exactly solvable, [7], since it preserves the subspace $\mathcal{N} \subset C^{\infty}\left(\mathbb{R}^{2}\right)$ whose elements are the polynomials in the variables $(x, y)$ of total degree less than or equal to 2 .

Let $J$ denote the following element of the universal enveloping algebra of $\mathfrak{g}$ :

$$
\begin{aligned}
J=\left(J^{1}\right)^{2} & +\left(J^{2}\right)^{2}+4(a-b)\left(J^{3}\right)^{2}+4 b\left(J^{5}\right)^{2}+4 a(a-2 b)\left(J^{6}\right)^{2} \\
& +2 a\left\{J^{3}, J^{5}\right\}-4(2 a+b) J^{3}+2(a-12 b) J^{5}+5(7 b-a),
\end{aligned}
$$

where $a, b$ are real parameters such that

$$
a>2 b>0 .
$$

If we define

$$
\sigma=\frac{1}{4} \log \left[1+2(a-2 b) x^{2}\right]-\frac{3}{2} \log \left[1+2\left(a x^{2}+2 b y^{2}\right)\right],
$$

then it can be shown that

$$
-e^{\sigma} \cdot J \cdot e^{-\sigma}=h,
$$

where $h=-\Delta+V(x, y)$ is a Schrödinger operator on the manifold $M=\mathbb{R}^{2}$ endowed with an appropriate metric. More precisely (cf. [7], Example 4.3.3), the contravariant metric tensor $\left(g^{i j}\right)$ has components

$$
\begin{aligned}
& g^{11}=\left(1+2 a x^{2}\right)\left[1+2(a-2 b) x^{2}\right], \\
& g^{12}=2 a x y\left[1+2(a-2 b) x^{2}\right], \\
& g^{22}=1+4 b y^{2}+4 a(a-2 b) x^{2} y^{2},
\end{aligned}
$$

and Gaussian curvature

$$
\left.K=-2 a\left[1+4(a-2 b) x^{2}\right)\right],
$$

and the potential $V(x, y)$ is given by

$$
V=-3 a(a-2 b) x^{2}-\frac{b}{1+2(a-2 b) x^{2}}-\frac{48 b}{1+2\left(a x^{2}+2 b y^{2}\right)} .
$$


Since $J$ belongs to the universal enveloping algebra of $\mathfrak{g}$ by construction, it restricts to the finite-dimensional vector space $\mathcal{N}$. We can therefore easily diagonalize $J \mid \mathcal{N}$, obtaining the eigenvalues

$$
\begin{gathered}
\lambda_{0}=-5 a+23 b+4 s, \quad \lambda_{1}=-5 a+23 b-4 s, \quad \lambda_{2}=-a+3 b, \\
\lambda_{3}=-9 a+27 b, \quad \lambda_{4}=-3 a+15 b, \quad \lambda_{5}=-3 a+7 b,
\end{gathered}
$$

where

$$
s=\sqrt{a^{2}-2 a b+9 b^{2}} .
$$

Their corresponding eigenfunctions are given by

$$
\begin{aligned}
& \varphi_{0}=3 b+s+2 a(a-2 b) x^{2}, \quad \varphi_{1}=3 b-s+2 a(a-2 b) x^{2}, \\
& \varphi_{2}=1+2 a x^{2}-16 b y^{2}, \quad \varphi_{3}=x, \quad \varphi_{4}=y, \quad \varphi_{5}=x y .
\end{aligned}
$$

By (42), the Schrödinger operator $h$ possesses the six eigenfunctions

$$
\psi_{i}=e^{\sigma} \varphi_{i}, \quad 0 \leq i \leq 5,
$$

with energies

$$
E_{i}=-\lambda_{i} .
$$

The first of these eigenfunctions has no zeros, and therefore it must correspond to the ground state of $h$. The ground state energy of $h$ is then given by

$$
E_{0}=5 a-23 b-4 s
$$

which is indeed manifestly lower than the remaining five energies (43) by (41).

To define the twisted Hamiltonian $H$ and the Darboux transform of the eigenfunctions $\psi_{i}$, it is convenient to take as $e^{-\chi}$ a constant multiple of the ground state $\psi_{0}$, since this eigenfunction has no zeros. We shall therefore define

$$
\chi=-\log \left(x^{2}+c\right)-\sigma,
$$

where the constant $c>0$ is given by

$$
c=\frac{3 b+s}{2 a(a-2 b)} .
$$

According to (29), the action of $H$ on a vector field $X$ can be expressed as

$$
\left(\hat{H}_{1} X\right)^{i}=\left(-\nabla_{j} \nabla^{j}+V_{1}\right) X^{i}+V^{i}{ }_{j} X^{j},
$$


where $V_{1}=V-E_{0}+K$ and $V^{i}{ }_{j}=2 \nabla^{i} \nabla_{j} \chi$. After a long but straightforward calculation we find that

$$
\begin{gathered}
V_{1}=-7 a+23 b+4 s-11 a \gamma x^{2}-\frac{b}{1+2 \gamma x^{2}}-\frac{48 b}{1+2\left(a x^{2}+2 b y^{2}\right)}, \\
V_{2}^{1}=-\frac{48 a b x y\left(1+2 \gamma x^{2}\right)}{1+2\left(a x^{2}+2 b y^{2}\right)}, \\
V_{1}^{2}=-\frac{48 a x y\left(b+a \gamma x^{2}\right)}{1+2\left(a x^{2}+2 b y^{2}\right)}+\frac{x y P_{6}(x)}{\gamma a\left(c+x^{2}\right)^{2}\left(1+2 \gamma x^{2}\right)},
\end{gathered}
$$

where

$$
\gamma=a-2 b>0
$$

and

$$
\begin{gathered}
P_{6}(x)=48 a^{2} \gamma^{3} x^{6}+4 a \gamma^{2}(9 a+12 b+4 s) x^{4}+4 \gamma\left(5 a^{2}-9 a b-a s+18 b s+54 b^{2}\right) x^{2} \\
+5 a^{2}-22 a b-4 a s+30 b s+90 b^{2} .
\end{gathered}
$$

Note, in particular, that $V_{2}^{1} \neq V^{2}$.

The Darboux transforms $d^{-} \psi_{i}$ of the eigenfunctions $\psi_{i}, 1 \leq i \leq 5$, constructed above can also be computed in a straightforward way using (31), with the following result:

$$
\begin{aligned}
& d^{-} \psi_{1}=\frac{4 e^{\sigma} s x}{c+x^{2}} d x \\
& d^{-} \psi_{2}=2 e^{\sigma}\left[\frac{x\left(16 b y^{2}+2 a c-1\right)}{c+x^{2}} d x-16 b y d y\right] \\
& d^{-} \psi_{3}=e^{\sigma} \frac{c-x^{2}}{c+x^{2}} d x \\
& d^{-} \psi_{4}=e^{\sigma}\left[-\frac{2 x y}{c+x^{2}} d x+d y\right] \\
& d^{-} \psi_{5}=e^{\sigma}\left[\frac{y\left(c-x^{2}\right)}{c+x^{2}} d x+x d y\right] .
\end{aligned}
$$

As explained in Section 4, the five vector fields associated to these one-forms are formal eigenvectors of $\hat{H}_{1}$ with eigenvalues $E_{i}-E_{0}, 1 \leq i \leq 5$. For example, the first of these vector fields is

$$
4 s x \frac{1+2 \gamma x^{2}}{c+x^{2}} e^{\sigma}\left[\left(1+2 a x^{2}\right) \partial_{x}+2 a x y \partial_{y}\right] \text {. }
$$

In this case, it is easy to show that the five formal eigenvectors of $\hat{H}_{1}$ constructed above are actually square-integrable. Indeed, the square $\mathrm{L}^{2}$ norm of a vector field $X$ can be expressed as

$$
\|X\|^{2}=\int_{\mathbb{R}^{2}} \sqrt{g} g^{i j} X_{i} X_{j} d x d y
$$


where $g=\operatorname{det}\left(g_{i j}\right)$ and $X_{i}(i=1,2)$ are the components of the associated one-form $X^{b}$. But

$$
e^{2 \sigma} \sqrt{g}=\left(1+2 a x^{2}+4 b y^{2}\right)^{-7 / 2}<\text { const. } r^{-7}, \quad r=\sqrt{x^{2}+y^{2}} \rightarrow \infty,
$$

and a straightforward calculation shows that $e^{-2 \sigma} g^{i j} X_{i} X_{j}$ is bounded by a constant times $r^{4}$ as $r \rightarrow \infty$ when $X^{b}=d^{-} \psi_{i}$ for $i=1, \ldots, 5$, thus proving our contention.

The limiting case

$$
a=2 b
$$

of the previous example is worth studying, because in this case the curvature is constant and negative:

$$
K=-4 b<0 .
$$

Diagonalizing again $J \mid \mathcal{N}$ we find the following eigenvalues

$$
\lambda_{0}=25 b, \quad \lambda_{1}=9 b, \quad \lambda_{2}=b,
$$

of respective multiplicities 1,2 and 3 . The corresponding eigenfunctions are

$$
\varphi_{0}=1, \quad \varphi_{1}=x, \quad \varphi_{2}=y, \quad \varphi_{3}=x y, \quad \varphi_{4}=12 b x^{2}-1, \quad \varphi_{5}=12 b y^{2}-1
$$

As before, the six functions $\psi_{i}=e^{\sigma} \varphi_{i}$ are eigenfunctions of $h$ with eigenvalue $E_{i}=-\lambda_{i}$, where $\sigma$ can be taken as

$$
\sigma=-\frac{3}{2} \log \left(1+4 b r^{2}\right)
$$

Again, the first of these eigenfunctions has no zeros, and therefore corresponds to the ground state, with ground energy

$$
E_{0}=-25 b
$$

Taking $\psi_{0}=e^{-\chi}$, or equivalently

$$
\chi=-\sigma
$$

we find

$$
V_{1}=20 b-\frac{48 b}{1+4 b r^{2}}, \quad V_{2}^{1}=V_{1}^{2}=-\frac{96 b^{2} x y}{1+4 b r^{2}} .
$$

The Darboux transforms of the eigenfunctions $\psi_{i}(1 \leq i \leq 5)$ are easily computed. Their associated vector fields, which are as usual eigenvectors of $\hat{H}_{1}$ with eigenvalues $E_{i}-E_{0}$, are

$$
\begin{array}{ll}
e^{\sigma}\left[\left(1+4 b x^{2}\right) \partial_{x}+4 b x y \partial_{y}\right], & e^{\sigma}\left[4 b x y \partial_{x}+\left(1+4 b y^{2}\right) \partial_{y}\right], \\
e^{\sigma}\left[\left(1+8 b x^{2}\right) y \partial_{x}+\left(1+8 b y^{2}\right) x \partial_{y}\right], & 24 b e^{\sigma}\left[\left(1+4 b x^{2}\right) x \partial_{x}+4 b x^{2} y \partial_{y}\right], \\
24 b e^{\sigma}\left[4 b x y^{2} \partial_{x}+\left(1+4 b y^{2}\right) y \partial_{y}\right] . &
\end{array}
$$

Since

$$
e^{2 \sigma} \sqrt{g}=\left(1+4 b r^{2}\right)^{-7 / 2}
$$


an argument analogous to the one for the previous example shows that the latter eigenvectors are all square integrable.

Consider now the canonical form 2.3 (with $n=2$ ) in the classification of reference [9], which is the Lie algebra $\mathfrak{g} \simeq \mathfrak{s l}(3, \mathbb{R})$ spanned by the first-order differential operators

$$
\begin{gathered}
J^{1}=\partial_{x}, \quad J^{2}=\partial_{y}, \quad J^{3}=x \partial_{x}, \quad J^{4}=y \partial_{x}, \quad J^{5}=x \partial_{y}, \quad J^{6}=y \partial_{y}, \\
J^{7}=x^{2} \partial_{x}+x y \partial_{y}-2 x, \quad J^{8}=x y \partial_{x}+y^{2} \partial_{y}-2 y .
\end{gathered}
$$

The latter Lie algebra leaves invariant the finite-dimensional subspace $\mathcal{N} \subset C^{\infty}\left(\mathbb{R}^{2}\right)$ introduced in the previous examples, so that it is again quasi-exactly solvable. Let $J$ denote the element of the universal enveloping algebra of $\mathfrak{g}$ given by

$$
J=a\left[\left(J^{1}\right)^{2}+\ell\left(J^{2}\right)^{2}\right]+c\left[\ell\left(J^{7}\right)^{2}+\left(J^{8}\right)^{2}\right]+b\left(\left\{J^{1}, J^{7}\right\}+\left\{J^{2}, J^{8}\right\}+7 J^{3}+7 J^{6}\right),
$$

where $a, c, \ell$ are positive real parameters, $b \geq 0$, and

$$
\gamma=\ell a c-b^{2}>0
$$

If

$$
\rho=b+c\left(\ell x^{2}+y^{2}\right)
$$

and

$$
\sigma=-\frac{5}{8} \log \left(\rho^{2}+\gamma\right)+\frac{7}{4} b \gamma^{-1 / 2} \arctan \left(\gamma^{-1 / 2} \rho\right)
$$

it is shown in [7] that (42) defines again a Schrödinger operator $h$, with potential $V$ given by

$$
V=-\frac{71}{4} b-\frac{3}{4} \rho+\frac{\left(192 b^{2}-45 a c \ell\right) \rho+143 a b c \ell-192 b^{3}}{4\left(\rho^{2}+\gamma\right)} .
$$

The metric of the Riemannian manifold $M=\mathbb{R}^{2}$ has now contravariant components

$$
\begin{aligned}
& g^{11}=a+x^{2}(b+\rho), \\
& g^{12}=x y(b+\rho), \\
& g^{22}=\ell a+y^{2}(b+\rho),
\end{aligned}
$$

and Gaussian curvature given by

$$
K=-2 \rho .
$$

The eigenvalues of $J \mid \mathcal{N}$ are easily found to be

$$
\lambda_{0}=4 b+2 s, \quad \lambda_{1}=4 b-2 s, \quad \lambda_{2}=12 b, \quad \lambda_{3}=2 b,
$$

where

$$
s=\sqrt{16 b^{2}+2 a c \ell}
$$


and the last two eigenvalues have multiplicity 2. The corresponding eigenfunctions are respectively

$$
\begin{aligned}
& \varphi_{0}=\ell x^{2}+y^{2}+\frac{s-4 b}{c}, \quad \varphi_{1}=\ell x^{2}+y^{2}-\frac{s+4 b}{c}, \\
& \varphi_{2}=\ell x^{2}-y^{2}, \quad \varphi_{3}=x y, \quad \varphi_{4}=x, \quad \varphi_{5}=y .
\end{aligned}
$$

On account of (42), multiplying these eigenfunctions of $J$ by the factor $e^{\sigma}$ we obtain six eigenfunctions $\psi_{i}=e^{\sigma} \varphi_{i}$ of $h$, with energies $E_{i}=-\lambda_{i}$. As in the previous examples, the first of these eigenfunctions never vanishes, and therefore it corresponds to the ground state of $h$, with ground energy given by

$$
E_{0}=-4 b-2 s .
$$

We therefore take

$$
\chi=-\log \psi_{0}=-\sigma-\log \left(\ell x^{2}+y^{2}+k\right)
$$

with

$$
k=\frac{s-4 b}{c}>0
$$

obtaining

$$
V_{1}=2 s-\frac{55}{4} b-\frac{11}{4} \rho+\frac{\left(192 b^{2}-45 a c \ell\right) \rho+143 a b c \ell-192 b^{3}}{4\left(\rho^{2}+\gamma\right)}
$$

and

$$
V_{2}^{1}=\frac{2 c x y P_{3}(\rho)}{\left(\rho^{2}+\gamma\right)(\rho+s-5 b)^{2}}, \quad V_{1}^{2}=\ell V_{2}^{1}
$$

where

$$
\begin{aligned}
P_{3}(\rho)=(27 b & -4 s) \rho^{3}+\left(9 a c \ell+14 b s-79 b^{2}\right) \rho^{2} \\
+ & \left(317 b^{3}-76 b^{2} s-16 a b c \ell+6 a c \ell s\right) \rho \\
& -201 b^{4}+187 a b^{2} c \ell-50 b \gamma s+14 a^{2} c^{2} \ell^{2} .
\end{aligned}
$$

Note that the denominator of $V^{1}{ }_{2}$ never vanishes on account of (44)-(45).

The Darboux transforms of the eigenfunctions $\psi_{i}(1 \leq i \leq 5)$ are given by

$$
\begin{aligned}
& d^{-} \psi_{1}=\frac{4 s e^{\sigma}}{c\left(k+\ell x^{2}+y^{2}\right)}(\ell x d x+y d y) \\
& d^{-} \psi_{2}=\frac{2 e^{\sigma}}{k+\ell x^{2}+y^{2}}\left[\ell x\left(k+2 y^{2}\right) d x-\left(k+2 \ell x^{2}\right) y d y\right] \\
& d^{-} \psi_{3}=\frac{e^{\sigma}}{k+\ell x^{2}+y^{2}}\left[y\left(k-\ell x^{2}+y^{2}\right) d x+x\left(k+\ell x^{2}-y^{2}\right) d y\right], \\
& d^{-} \psi_{4}=\frac{e^{\sigma}}{k+\ell x^{2}+y^{2}}\left[\left(k-\ell x^{2}+y^{2}\right) d x-2 x y d y\right] \\
& d^{-} \psi_{5}=\frac{e^{\sigma}}{k+\ell x^{2}+y^{2}}\left[-2 \ell x y d x+\left(k+\ell x^{2}-y^{2}\right) d y\right]
\end{aligned}
$$


The vector fields associated to these one-forms are eigenvectors of $\hat{H}_{1}$ with eigenvalue $E_{i}-E_{0}$. As in the previous examples, the latter vector fields are easily found to be square integrable on $M$ on account of the asymptotic behavior at infinity of $e^{2 \sigma} \sqrt{g}$ and $e^{-\sigma}\left(d^{-} \psi_{i}\right)$.

\section{Acknowledgments}

One of the authors (A.G.-L.) would like to thank A. Galindo, M. Mañas and M. A. MartínDelgado for helpful conversations.

\section{References}

[1] Andrianov, A. A., Borissov, N. V., and Ioffe, M. V., The factorization method and quantum systems with equivalent energy spectra, Phys. Lett. 105A (1984), 19-22.

[2] Andrianov, A. A., Borissov, N. V., Eides, M. I., and Ioffe, M. V., Supersymmetric origin of equivalent quantum systems, Phys. Lett. 109A (1985), 143-148.

[3] Crum, M. M., Associated Sturm-Liouville equations, Q. J. Math. 6 (1955), 121-127.

[4] Darboux, G., Théorie générale des surfaces, vol. II, Gauthier-Villars, Paris, 1888.

[5] Deift, P., and Trubowitz, E., Inverse scattering on the line, Comm. Pure Appl. Math. 32 (1979), 121-251.

[6] Goursat, E., Leçons sur l' intégration des equations aux dérivées partielles du second ordre, Hermann, Paris, 1896.

[7] González-López, A., Kamran, N., and Olver, P.J., New quasi-exactly solvable Hamiltonians in two dimensions, Commun. Math. Phys. 159 (1994), 503-537.

[8] González-López, A., Kamran, N., and Olver, P.J., Quasi-exact solvability, Contemp. Math. 160 (1994), 113-140.

[9] González-López, A., Kamran, N., and Olver, P.J., Real Lie algebras of differential operators and quasi-exactly solvable potentials, Phil. Trans. London Math. Soc. A354 (1996), 1165-1193.

[10] Infeld, L., and Hull, T., The factorization method, Revs. Mod. Phys. 23 (1951), $21-68$.

[11] Kamran, N., and Tenenblat, K., Laplace transformation in higher dimensions, Duke Mathematical Journal 84 (1996), 237-266 .

[12] Moutard, Th. F., Sur la construction des équations de la forme $z^{-1} z_{x y}=\lambda(x, y)$, qui admettent une intégrale générale explicite, J. de L'Ecole Polytech. Cahier 45 (1878), 834.

[13] Ushveridze, A., Quasi-exactly solvable models in quantum mechanics, IOP, Bristol, 1994.

[14] Vassiliou, P. J., On some geometry associated with a generalised Toda lattice, Bull. Austral. Math. Soc. 49 (1994), 439-462.

[15] Veselov, A. P., and and Novikov, S. P., Exactly solvable periodic two-dimensional Schrödinger operators, Uspekhi Mat. Nauk 50 (1995), 171-172. 
[16] Witten, E., Dynamical breaking of supersymmetry, Nucl. Phys. B188 (1981), $513-555$.

[17] Witten, E., Supersymmetry and Morse theory, J. Diff. Geom. 17 (1982), 661-692. 